\title{
Dual diagnosis: management within a psychosocial context
}

\author{
Mohammed T. Abou-Saleh
}

\begin{abstract}
Recent developments in UK government policy have highlighted the unmet needs of people with dual diagnosis (comorbidity of substance misuse and psychiatric disorder, particularly severe mental illness). Advances in assessment techniques and diagnostic practice have informed the treatment of comorbidity and improved its outcome. There is growing evidence for the effectiveness of psychosocial interventions such as motivational interviewing and cognitive-behavioural therapy, mostly from US studies. However, within the secondary care provided by addiction and general psychiatric services there are serious implementation barriers related to service organisation, staffing levels, training and - most importantly - the difficulties of engaging people with severe mental illness and comorbid substance misuse in treatment. The evidence for the effectiveness of psychosocial treatments and models of service is reviewed and challenges for optimal practice in the UK are highlighted.
\end{abstract}

This article is the first in a short series addressing issues relating to dual diagnosis. The next to be published (Crome E Myton, 2004) will discuss pharmacotherapeutic interventions in dual diagnosis.

The co-occurrence of substance misuse with other psychiatric disorders is increasingly recognised as a major public health problem. The term 'dual diagnosis' has been introduced to describe this phenomenon, but 'comorbidity' might be a better term. Community-based surveys in the USA and the UK have reported high rates of comorbidity, particularly substance misuse in people with serious mental illness (Harrison \& Abou-Saleh, 2002). Farrell et al (2001), in a national household survey, reported prevalence rates of psychiatric disorder of $22 \%$ in nicotine dependence, $30 \%$ in alcohol dependence and $45 \%$ in drug dependence, compared with $12 \%$ prevalence in the non-dependent population.

The pattern of this comorbidity varies between comorbid mood, anxiety and personality disorders in patients accessing addiction services, and comorbid alcohol, cannabis and cocaine misuse in patients accessing general psychiatric services (Abou-Saleh, 2000). Comorbidity is associated with increased risk of violence, suicide and worse clinical and social outcomes. The National Confidential Inquiry into Suicide and Homicide has reported substance misuse as a factor in over half of homicides and suicides by people with serious mental illness (Department of Health, 2001). Moreover, this group has high rates of criminality and blood-borne infections, including HIV infection and hepatitis B and C.

The severity of this morbidity also varies between these specialist settings: severe psychiatric disorder is associated with non-dependent use of substances (problematic substance misuse), whereas severe addiction is associated with personality disorder with or without minor psychiatric disorder. The pattern and severity of this comorbidity are therefore related to the clinical setting in which it presents, which may not be representative of comorbidity occurring in the community.

My aim in this article is to describe the policy context, define dual diagnosis, identify meaningful subgroups and describe key aspects of their assessment and treatment, with emphasis on psychosocial approaches in the context of integrated service models.

\section{Policy context}

The National Service Framework (NSF) for Mental Health (Department of Health, 1999), while emphasising the importance of tackling dual

Mohammed T. Abou-Saleh is a reader and honorary consultant in addiction psychiatry in the Department of Mental Health, St George's Hospital Medical School, University of London, and Clinical Director of Addiction Services and Director of Research and Development in South West London and St George's Mental Health Trust (Cranmer Terrace, London SW17 0RE, UK. Tel: 0208725 0368; fax: 0208725 2914; e-mail: mabousal@sghms.ac.uk). He was previously Professor and Chairman of the Department of Psychiatry and Behavioural Sciences, Faculty of Medicine and Health Sciences, United Arab Emirates University and a reader in psychiatry at the University of Liverpool. His research interests include biological psychiatry, dual diagnosis, addiction prevention and psychopharmacology. 
diagnosis, failed to include standards and service models to address the challenges posed by patients with dual diagnosis, including those with severe mental illness. It was in the context of this gap in policy that the Dual Diagnosis Good Practice Guide (Department of Health, 2002a) was launched. The NSF also omitted to provide standards and service models for people with substance use disorders, an omission that was addressed by the complementary guidance on models of care launched by the National Treatment Agency for Substance Misuse (2002).

The key message in the Dual Diagnosis Good Practice Guide is that substance misuse is usual rather than exceptional among people with severe mental health problems, and that the relationship between the two disorders is complex. Individuals with dual diagnosis have varied and complex needs and require high-quality, comprehensive and integrated care that should be delivered within the mainstream mental health services. The guide recognises that mainstreaming will not reduce the role of drug and alcohol services, which will continue to treat the majority of people with substance misuse problems and to advise on substance misuse issues. The guide summarises good practice in relation to treatment and sets out a programme for local implementation of the appropriate service model. It is concluded that integrated care by a single team delivers better outcomes than serial care (sequential referrals to different services) or parallel care (more than one service engaging with a patient at the same time). The guide recommends that more UK-based research is required to establish the evidence base, and that well-organised parallel care can be used as a stepping stone to integrated treatment delivered by existing mental health services following training and support from substance misuse services.

It should be noted that the Dual Diagnosis Good Practice Guide was launched at the same time and place as two other guidelines: Adult Acute Inpatient Care Provision Guidance (Department of Health, 2002b) and National Minimum Standards for Psychiatric Intensive Care Units and Low Secure Environments (Department of Health, 2002c). Neither of these other publications emphasises the importance of dual diagnosis. The intensive care guidance specifies substance misuse as an exclusion criterion on two counts, and falls into the trap of emphasising the primacy of one diagnosis over another in the acute situation, potentially perpetuating the passing of complex patients from 'pillar to post' - the title of a video produced by the mental health charity Mind in Croydon (2004) - because of the recognised diagnostic uncertainty and tautology. It is simplistic to adopt a dualistic approach to behaviour where the relationship between the two conditions is properly comorbid, with shared aetiological factors.

\section{Models of care}

The National Treatment Agency guidance on models of care provides a treatment framework and process intended to support the move towards consensus about the essential components of specialist substance misuse services and the importance of links with other health, social care and criminal justice agencies. The guidance structures substance misuse services into four broad tiers, from Tier 1 (non-specialist services, including general psychiatric services) to Tier 4(a), which offers residential care specifically for substance misusers, and Tier 4(b), highly specialised services unrelated to substance misuse, such as forensic psychiatric services and specialist personality disorder services. The guidance also stipulates the development of integrated care pathways as the preferred method of applying packages of care in a coordinated and integrated way. The integrated care pathways provide a means of agreeing local referral and treatment protocols to define where and when a particular service user needs to be referred.

\section{Treatment of personality disorder}

The new national guidance Personality Disorder: No Longer a Diagnosis of Exclusion (Department of Health, 2003) has highlighted the inadequate provision for people with personality disorders. This is related to the reluctance of clinicians to treat these people because they do not have the skills, the training or the resources. A survey of NHS trusts in England found that only $17 \%$ provide dedicated services for patients with personality disorders and $28 \%$ provide no identified service. The guidance aims to increase specialist provision for patients with personality disorders within mainstream and specialist psychiatric services, including forensic and addiction psychiatry, and to introduce the necessary education and training of mental health practitioners in these services. In view of the common occurrence of alcohol and drug misuse in patients with personality disorders, referral pathways and protocols need to be agreed between general, forensic and addiction services for the provision of treatment, training and support.

\section{Defining dual diagnosis}

Currently recognised subgroups of patients with dual diagnosis are defined by presumed aetiological mechanisms: primary psychiatric disorder with secondary substance misuse, substance misuse with secondary psychiatric disorder, and psychiatric symptoms related to substance intoxication 
or withdrawal. These categories are consistent with the operationally defined corresponding categories in DSM-IV (American Psychiatric Association, 1994), in which a distinction is made between independent (primary psychiatric comorbidity) and substance-induced (organic) psychiatric comorbidity and the category of expected symptoms of substance use or withdrawal. The evidence base for these aetiological subgroups has identified two key aetiological factors for dual diagnosis: the presence of underlying antisocial personality disorder and the vulnerability of people with severe mental illness to non-dependent problematic substance misuse (the supersensitivity model; Mueser et al, 1998). The self-medication hypothesis has not been sustained despite its plausibility and popularity with users and clinicians alike. In the case of schizophrenia, it has been suggested that the emergence of comorbid substance misuse is integral to the disorder and is related to the disconnection in the neural networks that occurs in the schizophrenia disease process (Chambers et al, 2001). An important piece of the jigsaw that is often missing is the underlying personality disorder, offering a triple diagnosis. This absence is related to the difficulties of reliably diagnosing personality disorder in the context of comorbidity. An inclusive and pragmatic approach would offer the typology of:

- severe psychiatric disorder with problematic substance misuse, with or without an underlying personality disorder;

- substance-induced psychiatric disorder;

- substance dependence with personality disorder, without comorbid psychiatric disorder.

\section{Assessment}

As comorbidity is the rule rather than the exception, it is crucial that all patients are routinely screened to detect substance misuse in those presenting to general psychiatric services and to detect psychiatric problems in those presenting to addiction services. Detection is based on self-report, informant report and laboratory tests. Hair analysis carries a major advantage over urine testing in that it covers much longer periods than a single urine test (McPhillips et $a l, 1997)$. Those with comorbidity are then provided with a specialised assessment (Noordsy et al, 2003), which should include the items listed in Box 1.

Diagnostic assessment of comorbidity optimally takes place following detoxification, to disentangle substance-induced disorders from primary comorbid psychiatric disorders and expected effects of substance intoxication/withdrawal using
Box 1 Essential assessments for patients with dual diagnosis (comorbidity)

- A detailed assessment of the substance misuse and its interaction with the psychiatric disorder

- A functional assessment of the impact of substance misuse on relationships, housing, work, leisure and personal goals

- Assessment of risks of self-harm, harm to others, self-neglect and blood-borne viral infections

- Assessment of motivation for change and preferences for treatment

- Treatment planning to address motivation to reduce or stop substance misuse, and pressing needs including social needs, risk, medical and psychiatric conditions

DSM-IV criteria (see above). Psychiatric symptoms or disorder that persist after 4 weeks of substance withdrawal indicate primary comorbidity. Similar considerations apply for the optimal assessment of personality disorders (Axis I), particularly in the setting of Axis II comorbidity.

\section{Screening tests}

A number of screening instruments have been introduced to assess comorbidity (Box 2) (Crawford \& Crome, 2001), including the following.

- The Psychiatric Research Interview for Substance and Mental Disorders (PRISM) is a diagnostic interview based on DSM-IV. It is reported to be more reliable than previous instruments for assessing psychiatric disorders in those who have comorbid substance use disorders (Hasin et al, 1996).

- The Dartmouth Assessment of Lifestyle Instrument (DALI) is an 18-item intervieweradministered tool that takes on average 6 minutes to complete (Rosenberg et al, 1998). It was formed as a composite of the most validated questions from ten established screening questionnaires for substance use disorder. It can be used as a screening instrument for substance use disorder in the psychiatric population. It was developed primarily to detect alcohol, cannabis and cocaine use disorders. The DALI is available at http: / / www.dartmouth.edu/psychrc/ pdf_files/DALI.pdf.

- The Alcohol Use Disorders Identification Test (AUDIT; Babor et al, 1992) is a brief self-report 
Box 2 Assessment and screening tests (based on Crawford \& Crome, 2001)

A thorough and ongoing assessment, which includes a comprehensive history, should underpin comorbidity treatment

- When diagnostic issues are complex, the improved reliability of the PRISM is worth the extra effort

- The DALI may be useful for assessing alcohol, cannabis and cocaine use disorders in people with severe mental illness

- The CUAD is potentially useful for detecting substance use disorders in severely mentally ill patients

- The SATS can be used to evaluate treatment progress or as an outcome measure

- Neurocognitive impairment will affect the assessment process

- Biochemical screening increases rates of identification of substance use

- Hair analysis covers much longer periods than a single urine specimen

questionnaire developed by the World Health Organisation to identify people whose alcohol consumption has become hazardous or harmful to their health. The questions asked (about consumption and problems associated with dependence and regular use or intoxication also enable practitioners to identify areas that require attention.

- The Drug Abuse Screening Test (DAST-10) (Skinner, 1982) is a self-report questionnaire for measuring the severity of drug (not alcohol) dependence.

- The Mini-Mental State Examination (MMSE; Folstein et al, 1975) is a brief, quantitative measure of cognitive status in adults. It can be used to screen for cognitive impairment, to estimate its severity, to follow cognitive changes over time and to document response to treatment.

- The Chemical Use, Abuse and Dependence (CUAD) scale is a brief (20 minutes to administer), reliable and validated tool for the identification of substance use disorders in severely mentally ill in-patients (Appleby et al, 1996).

- The Substance Abuse Treatment Scale (SATS; McHugo et al, 1995) can be used to evaluate treatment progress or as an outcome measure. The scale is intended for assessing a person's stage of substance misuse treatment.

\section{Treatment models}

People with comorbidity often fall into the cracks between general psychiatric and specialist addiction services. Whereas the culture of addiction services is dedication to 'clients' who want to be helped, general psychiatric services are obliged to treat all patients, including reluctant ones detained under the Mental Health Act. The plight of these patients is compounded by the lack of community care facilities, including residential care, while day centres and rehabilitation facilities often do not admit them. Several models for treatment of comorbidity have evolved.

The serial treatment model involves management of the psychiatric disorder and substance misuse in separate settings and services; for example, patients are treated first in the general psychiatric service, and when recovered are referred to the specialist addiction service.

The parallel treatment model involves the concurrent treatment of substance misuse and psychiatric disorder by different staff and in different settings. Both serial and parallel treatment models have serious limitations for optimal treatments of both disorders, with high rates of patient withdrawal.

The preferred model is integrated treatment, in which the same staff treat both disorders in the same setting. The limitation of this model, however, is that it may not provide the same intensity of treatment for the substance misuse as is afforded to the psychiatric disorder. A review by Drake et al (1998) of 36 studies of the effectiveness of integrated treatment for dual diagnosis patients identified ten studies (six uncontrolled and four controlled) of comprehensive, integrated out-patient treatment programmes which were effective in engaging patients in services, reducing substance use and sustaining remission. Outcomes related to hospital use, psychiatric symptoms and other domains were less consistent. Several features of the programmes appeared to be associated with effectiveness: assertive outreach, case management, and a longitudinal, stage-wise, motivational approach to treatment of the substance misuse.

The integrated approach involves individually tailored treatments and differs from traditional substance misuse treatment in several respects:

- the focus is on preventing anxiety, rather than breaking through denial;

- it emphasises trust, understanding and learning, rather than confrontation and criticism;

- it emphases reducing harm from substance use, rather than abstinence; 
Box 2 Principles of treatment of substance misuse in people with severe mental illness (Drake et al, 1993)

- Assertive outreach to facilitate engagement

- Close monitoring to provide structure and social reinforcement

- Integrated concurrent service

- Comprehensive, wide range of interventions

- Stable living situation

- Flexibility and specialisation (modified approaches)

- Stages of treatment: engagement, persuasion, active treatment and relapse prevention

- Longitudinal perspective for relapsing and chronic disorder

- Optimism - instilling hope in patients and carers

- it uses slow-paced and long-term management, rather than rapid withdrawal and short-term treatment;

- it provides staged and motivational counselling, rather than confrontation;

- it employs supportive clinicians readily available in familiar settings, rather than only during working hours and at clinics;

- 12-step (Alcoholics Anonymous) groups are available for those who choose and can benefit from them, rather than being required for all patients.

Drake et al (1993), using an evidence-based approach, identified nine principles for the treatment of substance misuse in severely mentally ill patients (Box 2).

A Cochrane systematic review (Ley et al, 1999) of treatment programmes for those with both severe mental illness and substance misuse identified six relevant studies. It found no clear evidence supporting an advantage over standard care of any type of programme, including integrated assertive community treatment and a residential treatment programme.

\section{Treatment considerations}

The treatment of comorbidity is fraught with difficulties which are related to diagnostic assumptions and to the setting in which the comorbidity is encountered. One consideration is a tendency for the comorbid condition to be considered as being of secondary importance and hence to be ignored or insufficiently treated. This is based on the assumption that the comorbid disorder is secondary to the primary disorder - be it substance misuse or other psychiatric disorder - and that treatment of the primary disorder may resolve the secondary disorder, which is not considered to require specific treatment. Also, there is general reluctance to treat psychiatric patients who misuse substances. Reasons for this are multiple, and include concern about possible toxic interaction between the prescribed medication and the substances that are misused; the assumption that active substance misuse will cause worsening of comorbid psychiatric symptoms and impair response to treatment; and the fear that 'enabling' the patient by treating the psychiatric illness would diminish the patient's motivation to deal with the substance misuse problem (Weiss \& Najavits, 1998).

Although assessment of comorbidity is best done after the substance of misuse has been withdrawn, complete withdrawal is rarely obtained in an out-patient setting and treatment of the psychiatric disorder often begins while the patient is still misusing the substance. There is evidence that treatment of the psychiatric disorder during active substance misuse is effective and occasionally also has a positive impact on the substance misuse itself. Saxon \& Calsyn (1995) showed that outcome at the end of 1 year of treatment in a substance misuse programme was as favourable for dually diagnosed patients as for those with substance misuse alone. Controlled trials of antidepressants in patients with depression who misuse alcohol have demonstrated efficacy in treatment of the depressive disorder and modest effect on the substance misuse. These findings counter the assumption that treatment of the comorbid psychiatric disorder in those actively engaged in substance misuse represents a form of 'enabling' (Cornelius et al, 1997).

\section{Specific treatment approaches}

Treatment is guided by a comprehensive assessment, and in integrated settings it involves a number of interventions matching patients' needs. Treatment is normally provided in the community with assertive outreach, but is also given in in-patient settings, particularly when the patient needs to be stabilised, to be assessed following detoxification and to achieve abstinence. Importantly, staff should hold a realistic and longitudinal view of treatment of substance misuse, with different interventions matched to different stages of the treatment process:

- Engagement - regular contact and development of a therapeutic alliance, and meeting basic needs.

- Persuasion - motivational techniques to enhance motivation to change (reduce substance use). 
Table 1 Potential interventions at different stages of treatment

Stage of treatment

Case management

Family work

Pharmacological treatment

Assertive outreach

Coerced or involuntary interventions

\begin{tabular}{cccc} 
& \multicolumn{2}{c}{ Stage of treatment } \\
Engagement & Persuasion & Active treatment & Relapse prevention \\
$\times$ & $\times$ & $\times$ & $\times$ \\
$\times$ & $\times$ & $\times$ & $\times$ \\
$\times$ & $\times$ & $\times$ & $\times$ \\
$\times$ & $\times$ & $\times$ & \\
$\times$ & $\times$ & $\times$ & \\
& $\times$ & $\times$ & \\
& $\times$ & $\times$ & \\
& $\times$ & $\times$ & $\times$ \\
& $\times$ & $\times$ & $\times$ \\
& $\times$ & $\times$ & $\times$ \\
& $\times$ & $\times$ & $\times$ \\
& $\times$ & $\times$
\end{tabular}

Motivational interviewing

Persuasion groups

Cognitive-behavioural counselling

Social skills training

Vocational rehabilitation

Active treatment groups

Self-help groups

From Noordsy et al (2003). () John Wiley \& Sons Ltd. Reproduced with permission.

- Active treatment - from harm reduction to abstinence-oriented approaches.

- Relapse prevention - identification of highrisk situations for relapse and management of future relapses.

Noordsy et al (2003) have mapped various interventions at different stages of treatment (Table 1 ).

Drake \& Mueser (2000) identified the following common components of integrated treatment.

- Case management - multidisciplinary case management with assertive outreach to engage and retain patients in community services.

- Close monitoring - medication supervision, urine drug screening and coercive approaches.

- Substance misuse treatment - motivational approaches; harm reduction and cognitivebehavioural therapy in individual, group and family settings; self-help (12-step programmes) and social skills training.

- Rehabilitation - provision of long-term support in the community, whether day care or residential care, to enable restoration of social and occupational function (supported education and employment).

- Housing - both supported and independent.

- Pharmacotherapy - provision of antipsychotic medication (particularly clozapine) in those with schizophrenia, and improvement of compliance by providing education and medication supervision.

\section{Evaluations of treatment interventions \\ General dual diagnosis}

In the USA, Drake et al (1998) in the context of their integrated treatment services conducted a randomised controlled trial of assertive community treatment in comparison with standard care in patients with dual diagnosis. Assertive community treatment (ACT) showed greater improvement in substance misuse and quality of life. Further analysis of the results showed that patients in highfidelity (with faithful implementation of and adherence to the ACT model) programmes showed greater reductions in alcohol and drug use and achieved higher rates of remission from substance misuse than those in low-fidelity programmes (McHugo et al, 1999).

\section{Dual diagnosis with bipolar disorder}

Studies in the USA have shown that integrated psychoeducation group therapy for in-patients is effective in improving comorbidity (Galanter et al, 1994). A trial of integrated group therapy with a focus on rapid intervention in patients with bipolar disorder and substance misuse showed greater efficacy in improving drug use, manic symptoms and medication compliance than non-integrated group therapy (Weiss et al, 2000).

\section{Dual diagnosis with psychosis}

In Australia, Kavanagh et al (2003) evaluated a brief intervention for substance misuse in early psychosis and showed that motivational interviewing during acute in-patient treatment was associated with reduction in substance misuse at 6-month and 12month follow-up compared with standard care.

In the UK, Barrowclough et al (2001) conducted a randomised controlled trial of family intervention in psychosis with substance misuse. The intervention, which comprised five weekly sessions of motivation interviewing, six sessions of cognitive therapy held every 2 weeks, and 10-16 sessions of family intervention was more effective than routine 
care in improving general functioning, symptoms and days of abstinence from substance misuse over 12 months. Another UK project, the Combined Psychosis and Substance Use (COMPASS) programme, has reported positive findings using cognitive-behavioural therapy techniques in the treatment of patients with dual diagnosis (Graham et al, 2003).

\section{Dual diagnosis with personality disorders}

The treatment of antisocial personality disorder by psychotherapy in patients with opiate addiction has yielded inconsistent results (Woody et al, 1985; Rounsaville et al, 1986). However, a study of methadone-treated patients with antisocial personality disorder reported that those who were rewarded quickly and frequently for not using drugs and were given progressively greater control over major aspects of their treatment had significantly lower rates of opiate and cocaine use than those who received standard treatment and who were only rewarded after they had achieved prolonged periods of abstinence (Brooner et al, 1998).

The national guidance on treatment of personality disorders (Department of Health, 2003) advocates a number of approaches, including scheme-based cognitive therapy, contingency management, dialectical behavioural therapy and the Henderson Hospital's therapeutic community approach. Although there is some evidence for the efficacy of these approaches, these treatments have not been evaluated in those with comorbid substance misuse except for a randomised controlled trial in patients with borderline personality disorder and substance misuse (van den Bosch et al, 2002). The study showed that dialectical behavioural therapy resulted in greater reductions in borderline symptoms and behaviours than treatment as usual, an effect that was not modified by the presence of comorbid substance misuse. Importantly, dialectical behavioural therapy had no effect on substance misuse, and it was advocated that specific treatment for substance misuse should be combined with dialectical behavioural therapy for dual therapeutic impact on borderline symptoms and substance misuse.

\section{Optimal services}

The optimal model of care for patients with comorbidity could be developed in the context of current service models and structures, provided minimum standards for quality are established (Abou-Saleh, 2000) (Box 3).

Johnson (1997) advocated the development of a dedicated, highly specialised service for patients with dual diagnosis along the lines of the integrated treatment model discussed above. She suggested integrated models of addiction workers working within community mental health teams (CMHTs); training and supervision of CMHT staff in substance misuse; and establishing dual diagnosis specialists in CMHTs. However, there is a risk that such a service could become too selective and exclusive, with two dangers: exclusion of the most difficult patients, and de-skilling of the staff working in addiction and general psychiatric services.

The national guidance on good practice in dual diagnosis (Department of Health, 2002a) is timely and welcome, and in so far as it focuses on the needs of patients with serious mental illness and comorbid substance misuse, it is a step in the right direction. It places lead responsibility with mainstream mental health services; provides 'joined-up thinking' at the policy level, standards of good practice in assessment and treatment, with good examples such as the Kingston Community Drug and Alcohol Team, the Haringey Dual Diagnosis Service and the COMPASS programme in Birmingham; and gives guidance on implementation and commissioning standards. However, the document fails to address important issues relating to social care, the resource implications of this major service development, and the interface between mainstream mental health services and addiction services, as well as implications for the future and the scope of addiction services.

Concerning social care, particularly the housing needs of this vulnerable population, there is a lack of provision, with limited access to mainstream community care and residential care in mental health and addiction. It is a prime task for local implementation teams working with drug action teams to tackle this problem. The provision of housing is also important because the pooled budget for drug misuse is targeted towards meeting the rising demand for drug misuse services, and it is doubtful whether mainstream mental health funding could cater for the needs of this population.

\section{Box3 Quality standards for service planning}

- Access to relevant services (crisis, support, housing, after-care, therapeutic and legal services)

- Responsive and flexible approaches (assessment, engagement, retention, managing chaos and crisis, individual responses)

- Continuous care and management (monitoring, liaison, involvement of carers, risk assessment and management)

- Adequately trained staff (access to mental health trained staff) 
A most important implication of the guidance on dual diagnosis is the role of addiction services visà-vis mainstream mental health services, which at present lack capacity to deal with this population. A survey of the training and support needs of staff working with patients with dual diagnosis showed that mental health service workers lacked the knowledge and skills for assessment and treatment of substance misuse and were insufficiently aware of the available resources and how to access substance misuse services (Maslin et al, 2001). The role of addiction services is paramount in providing training and support for development of capacity and, importantly, in sustaining this capacity with optimal supervision and the agreement of local shared care working arrangements and care pathways, including the care programme approach. Our experience at South West London and St George's Mental Health NHS Trust has been positive, with the introduction of shared-care working arrangements and protocols to ensure that patients with dual diagnosis who present to addiction services have optimum access to mental health services for assessment and treatment. However, one problem that has persisted is that of people with substance-induced psychiatric disorders who have a high risk of self-harm and harm to others and who are often not accepted by mainstream mental health services. From the perspective of addiction services, the introduction and implementation of the National Treatment Agency for Substance Misuse's (2002) models of care framework could address these difficulties. Commissioners and providers of services should consider how to integrate the guidance on dual diagnosis (Department of Health, 2002a) with the models of care framework to create a single policy document that also addresses referral to mental and addiction services from primary care.

\section{References}

Abou-Saleh, M. T. (2000) Substance misuse and comorbid psychiatric disorders. CPD Bulletin in Psychiatry, 2, 6167.

American Psychiatric Association (1993) Diagnostic and Statistical Manual of Mental Disorders (4th edn) (DSM-IV). Washington, DC: APA.

Appleby, L., Dyson, V., Altman, E., et al (1996) Utility of the chemical use, abuse, and dependence scale in screening patients with severe mental illness. Psychiatric Services, 47, 647-649.

Babor, T. F., de la Fuente, J. R., Saunders, J., et al (1992) AUDIT: The Alcohol Use Disorders Identification Test. Geneva: World Health Organization.

Barrowclough, C., Haddock, G., Tarrier, N., et al (2001) Randomized controlled trial of motivational interviewing, cognitive behavior therapy, and family intervention for patients with comorbid schizophrenia and substance use disorders. American Journal of Psychiatry, 158, 1706-1713.

Brooner, R. K., Kidorf, M., King, V. L., et al (1998) Preliminary evidence of good treatment response in antisocial drug abusers. Drug and Alcohol Dependence, 49, 249-260.
Chambers, R. A., Krystal, J. H. \& Self, D. W. (2001) A neurobiological basis for substance abuse comorbidity in schizophrenia. Biological Psychiatry, 50, 71-83.

Cornelius, J. R., Salloum, I. M., Cornelius, M. D., et al (1997) Fluoxetine in depressed alcoholics: a double blind placebocontrolled trial. Archives of General Psychiatry, 54, 700705.

Crawford, V. \& Crome, I. (2001) Co-existing Problems of Mental Health and Substance Misuse (Dual Diagnosis ): A Review of Relevant Literature. London: Royal College of Psychiatrists.

Crome, I. B. \& Myton, T. (2004) Pharmacotherapy in dual diagnosis. Advances in Psychiatric Treatment, 10, in press.

Department of Health (2001) Safety First: Five-Year Report of the National Confidential Inquiry Into Suicide and Homicide by People With Mental Illness. London: Department of Health.

Department of Health (2002a) Mental Health Policy Implementation Guide: Dual Diagnosis Good Practice Guide. London: Department of Health.

Department of Health (2002b) Adult Acute Inpatient Care Provision Guidance. London: Department of Health.

Department of Health (2002c) National Minimum Standards for Psychiatric Intensive Care Units and Low Secure Environments. London: Department of Health.

Department of Health (2003) Personality Disorder: No Longer a Diagnosis of Exclusion. London: Department of Health.

Drake, R. E., Bartels, S. J., Teague, G. B., et al (1993) Treatment of substance abuse in severely mentally ill patients. Journal of Nervous and Mental Disease, 181, 606-611.

Drake, R. E., McHugo, G. J., Clark, R. E., et al (1998) Assertive community treatment for patients with co-occurring severe mental illness and substance use disorder: a clinical trial. American Journal of Orthopsychiatry, 68, 00-00.

Drake, R. E., Mercer-McFadden, C. \& Mueser, K. T. (1998) Review of integrated mental health and substance abuse treatment for patients with dual disorders. Schizophrenia Bulletin, 24, 589-608.

Drake, R. E. \& Mueser, K. T. (2000) Psychosocial approaches to dual diagnosis. Schizophrenia Bulletin, 26, 105-117.

Edwards, J., Hinton, M., Elkins, L., et al (2003) Cannabis and first-episode psychosis: the Cap Project. In Substance Misuse in Psychosis: Approaches to Treatment and Service Delivery. Chichester: John Wiley \& Sons.

Farrell, M., Howes, S., Bebbington, P., et al (2001) Nicotine, alcohol and drug dependence and psychiatric comorbidity. Results of a national household survey. British Journal of Psychiatry, 179, 432-437.

Folstein, M. F., Folstein S. E. \& McHugh, P. R. (1975) 'MiniMental State': a practical method for grading the cognitive state of patients for the clinician. Journal of Psychiatric Research, 12, 189-198.

Galanter, M., Egelko, S., Edwards, H., et al (1994) A treatment system for combined psychiatric and addictive illness. Addiction, 89, 1227-1235.

Graham, H. L., Copello, A., Birchwood, M. J., et al (2003) Cognitive-behavioural integrated treatment approach for psychosis and problem substance use. In Substance Misuse in Psychosis: Approaches to Treatment and Service Delivery. Chichester: John Wiley \& Sons.

Harrison, C. \& Abou-Saleh, M. T. (2002) Psychiatric disorders and substance misuse psychopathology. In Dual Diagnosis, pp. 43-57. Oxford: Blackwell.

Hasin, D., Trautman, K., Miele, G., et al (1996) Psychiatric Research Interview for Substance and Mental Disorders (PRISM): reliability in substance abusers. American Journal of Psychiatry, 153, 1195-1201.

Johnson, S. (1997) Dual diagnosis of severe mental illness and substance misuse: a case for specialist services? British Journal of Psychiatry, 171, 205-208.

Kavanagh, D. J., Young, R., White, A., et al (2003) Start over and survive: a brief intervention for substance misuse in early psychosis. Substance Misuse in Psychosis: Approaches to Treatment and Service Delivery. Chichester: John Wiley \& Sons.

Ley, A., Jeffrey, D. P., McLaren, S., et al (1999) Treatment programmes for those with both severe mental illness and substance misuse. Cochrane Library, issue 4, 1-19. Oxford: Update Software. 
Maslin, J., Graham, H. L., Cawley, M., et al (2001) Combined severe mental health and substance use problems. What are the training and support needs of staff working with this client group? Journal of Mental Health, 10, 131-140.

McHugo, G. J., Drake, R. E., Burton, H. L., et al (1995) A scale for assessing the stage of substance abuse treatment in persons with severe mental illness. Journal of Nervous and Mental Disease, 183, 762-767.

McHugo, G. J., Drake, R. E., Teague, G. B., et al (1999) Fidelity to assertive community treatment and client outcomes in the New Hampshire dual disorders study. Psychiatric Services, 50, 818-824.

McPhillips, M. A., Kelly, F. J., Barnes, T. E., et al (1997) Detecting co-morbid substance abuse among people with schizophrenia in the community: a study comparing the results of questionnaires with analysis of hair and urine. Schizophrenia Research, 25, 141-148.

Mind in Croydon (2004) Pillar to Post. A Film about Dual Diagnosis. Video available from http://www. mindincroydon.org.uk/videos.asp

Mueser, K. T., Bellack, A. S. \& Blanchard, J. J. (1998) Comorbidity of schizophrenia and substance abuse: Implications for treatment. Journal of Consulting and Clinical Psychology, 60, 845-856.

Noordsy, D. L., McQuade, D. V. \& Mueser, K. T. (2003) Assessment considerations. In Substance Misuse in Psychosis Approaches to Treatment and Service Delivery (eds H. L. Graham, A. Copello, M. J. Birchwood, et al). Chichester: John Wiley \& Sons.

Rosenberg, S. D., Drake, R. E., Wolford, G. L., et al (1998) Dartmouth Assessment of Lifestyle Instrument (DALI): a substance use disorder screen for people with severe mental illness. American Journal of Psychiatry, 155, 232238.

Rounsaville, B. J., Kosten, T. R., Weissman, M. M., et al (1986) Prognostic significance of psychopathology in treated opiate addicts. A 2.5 year follow-up study. Archives of General Psychiatry, 43, 739-745.

Saxon, A. J. \& Calsyn, D. A. (1995) Effects of psychiatric care for dual diagnosis patients treated in a drug dependence clinic. American Journal of Drug and Alcohol Abuse, 21, 303-313.

Skinner, H. (1982) The Drug Abuse Screening Test. Addictive Behavior, 7, 363-371.

Van den Bosch, L. M., Verheul, R., Schippers, G. M., et al (2002) Dialectical behavior therapy of borderline patients with and without substance use problems. Implementation and long-term effects. Addictive Behavior, 27, 911-923.

Weiss, R. \& Najavits, L. (1998) Overview of treatment modalities for dual diagnosis patients: pharmacotherapy, psychotherapy, and 12-step programmes. In: Dual Diagnosis and Treatment: Substance Abuse and Comorbid Medical and Psychiatric Disorders. (eds H. Kranzler \& B. Rounsaville), p. 87. New York: Marcel Dekker.

Weiss, R. D., Griffin, M. L., Greenfield, S. F. et al (2000) Group therapy for patients with bipolar disorder and substance dependence: results of a pilot study. Journal of Clinical Psychiatry, 61, 361-367.

Woody, G. E., Luborsky, L., McLellan, A. T., et al (1985) Psychotherapy for opiate dependence. NIDA Research Monograph, 58, 9-29.

\section{MCQs}

1 Studies of dual diagnosis have shown that:

a the prevalence of substance misuse in psychotic disorders is of the order of $10 \%$

b psychotic disorders are most commonly associated with cannabis use

c there is a strong association with antisocial personality disorder

d substance-induced psychiatric disorders predominate

e there is a strong association with suicide.
2 Concerning national policies on the provision of services for people with dual diagnosis:

a the National Service Framework provides important guidance

b services should be provided within mainstream mental health services

c there is strong evidence for the effectiveness of integrated models of care in the UK

$\mathrm{d}$ addiction services have a prime role in the provision of training and supervision of staff

e models of care advocate integrated care pathways with enhanced care coordination for this population.

3 Practical clinical assessment of people with dual diagnoses includes:

a screening with the Chemical Use, Abuse and Dependence (CUAD) scale

b screening with the Alcohol Use Disorders Identification Test (AUDIT)

c administration of the Psychiatric Research Interview for Substance and Mental Disorders (PRISM)

d detailed assessment of substance misuse and its interaction with other psychiatric disorders and of risk behaviour

e analysis of hair rather than urine for drugs.

4 Treatment for substance misuse in severely mentally ill people includes:

a in-patient treatment as an essential component

b a long-term perspective for lapsing and chronic disorders

c close liaison with the criminal justice system

d the enhanced care programme approach

e community residential care and a stable living situation.

5 Concerning the treatment of people with dual diagnosis:

a motivation interviewing is a core approach

$b$ there is evidence for the efficacy of family therapy

c clozapine is particularly advocated for those with schizophrenia and comorbid alcohol misuse

d cognitive-behavioural techniques are optimally matched to the relapse prevention stage of treatment

e assertive outreach is a key principle. 